\title{
A Comment on the levels of antipsychotics and their metabolites in urine
}

\begin{abstract}
Opinion
Testing for patient compliance is important in mental health. Testing from blood samples has been the standard providing therapeutic drug monitoring while using urine test results for patient monitoring is a relatively recent option. ${ }^{1,2}$ As with any testing, the methods and the analytes evolve with time and equipment. From earlier studies that only looked for a small number of antipsychotics and perhaps blood evident metabolites in urine ${ }^{3}$ to later studies that afforded resolution and identification of as many as 58 antipsychotics and antidepressants in the same method, ${ }^{4}$ the use of urine samples continues to grow in this important medication monitoring application. Further, knowledge of quantitative levels of both parent drug and metabolites in urine evolves to provide more detailed information to the physician and improve patient monitoring. Such knowledge has led to the realization that applying blood resident analytes to urine testing of antipsychotics does not necessarily provide the most sensitive methods.
\end{abstract}

For example, urine testing for aripiprazole and its metabolite, dehydroaripiprazole, affords very low levels of either molecule in urine $^{5}$ while adding a novel metabolite that is only found in urine at relatively high levels can greatly improve the effective sensitivity of the test results. ${ }^{5,6}$ This letter is aimed at providing a brief review of both analytes and sample preparation methods that will provide the best effective sensitivity for monitoring antipsychotics in urine. This is accomplished through the review of very recent article by Kim et al. ${ }^{7}$ and their initial test results from a population of mental health patients identified for treatment through the legal system.

A recent paper regarding development and validation of a liquid chromatography/mass spectrometry mass spectrometry (LC/MSMS) method for the analysis of antipsychotics and their respective metabolites in urine was published in the Journal of Analytical Toxicology. ${ }^{7}$ This work by Kim, et al., was focused on assessing adherence of "mentally disordered probationers" to legally required antipsychotics. The authors note that the repercussions of "negative" test results could be severe and thus they have taken a very conservative approach to declaring negative results. This situation is the same as clinical patients who are prescribed antipsychotics and are monitored for their adherence to this paradigm by their physicians. Dosages for these drugs are set by medical practice as well as regulatory approvals. Again, the repercussions of negative test results can be serious. Any improvement in the effective sensitivity of the analytic method would benefit these patients.

The paper by Kim et al. ${ }^{7}$ is well organized with detailed descriptions of development and validation of a method for analysis of 25 antipsychotic drugs and metabolites in urine. The method does require sample pretreatment but is not overly long at 10 minutes run time. The analytes chosen for this method reflect those prescribed for the "probationers". In addition, the authors presented "probationer" data from as many as 112 subjects for one drug (Quetiapine) and 187 additional positive test results overall. In their words, "second generation antipsychotics such as Quetiapine, Risperidone, and Olanzapine and third generation antipsychotics such as Aripiprazole were most detected". ${ }^{7}$ In their table V, they present the data ranges,
Volume 10 Issue 3 - 202

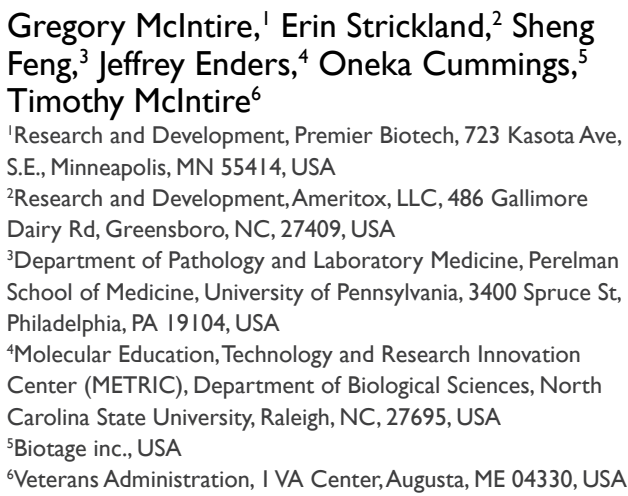

Correspondence: Gregory Mclntire, Research and Development, Premier Biotech, 723 Kasota Ave, S.E., Minneapolis, MN 554I4, USA, Email gregorymcintire2@gmail.com

Received: April 13, 202 | | Published: May 03, 202 |

Our first comment concerns the choices of metabolites for drugs such as aripiprazole (APZ) and Quetiapine (QTP). Our second comment relates to the impact of sample hydrolysis for drugs such as haloperidol (HPD) and quetiapine and its metabolite, 7 hydroxyquetiapine (HOQTP). Our third comment concerns the concentration ranges that are presented in the paper by Kim et al. ${ }^{7}$ relative to the earlier work by Feng et al. ${ }^{8}$

The choice of metabolites could have been improved. For example, aripiprazole (APZ) has been reported to metabolize to OPC $3373 .{ }^{5,6}$ Indeed, the metabolite, OPC 3373, was found approximately $50 \%$ more frequently than either aripiprazole or dehydroaripiprazole. ${ }^{5}$ Consistent with these reports, data in Table 1 demonstrate that neither APZ nor the metabolite, dehydroaripiprazole (DHAPZ), is as prevalent in the urine as the novel metabolite OPC 3373..$^{5,6}$ OPC 3373 is available commercially as a standard for such analysis. ${ }^{9}$ Strickland et al. ${ }^{9}$ reported that both the carboxylic acid and the sulfoxide of QTP were present at very high levels which were unaffected by hydrolysis. ${ }^{10}$ Again, the carboxylic acid of QTP is available commercially for these analyses. ${ }^{11}$ The impact of choosing a more prevalent metabolite for analysis is clearly demonstrated with the authors' choice of 7-hydroxychlorpromazine (HOCPZ) for analysis of chlorpromazine. This afforded a much wider data range than did chlorpromazine itself suggesting that the metabolite is more prevalent in urine than the parent drug. 
In several instances, the sensitivity of the method would have been enhanced via beta glucuronidase hydrolysis of various metabolites. For example, while the choice of 7-hydroxychlorpromazine was an advantage over the parent drug, it has been reported that the glucuronidated conjugate of 7-hydroxychlorpromazine is as much as 7 times more prevalent than the 7-hydroxylated metabolite itself. ${ }^{12}$ Thus, hydrolysis would have improved detection of HOCPZ in urine. Both HPD (e.g., Haldol) and QTP / HOQTP have been reported to be present as their respective glucuronide conjugates in urine. ${ }^{13-15}$ The HPD data in Table 1 from Feng et al. ${ }^{8}$ is hydrolyzed data and clearly demonstrates much higher values than those reported by Kim et al. ${ }^{7}$ and thus greater prevalence in urine than the parent drug itself. Although the data in Table 1 for QTP and HOQTP are from samples that were not hydrolyzed, the work of Cummings,${ }^{14}$ clearly demonstrates that hydrolysis would greatly add to the sensitivity of this method.

Table 1 shows the data from both papers. Feng et al. ${ }^{8}$ presents data from a much higher number of samples that were curated to report only those patient samples that were prescribed the drug and tested positive for the drug. ${ }^{8}$ In every case, the median value for a drug or its metabolite was higher in the report by Feng et al. ${ }^{8}$ in some cases much higher, than those reported by Kim et al. ${ }^{7}$ In every case, the range reported in Kim et al. ${ }^{7}$ is smaller than that reported by Feng et al. ${ }^{8}$ The olanzapine / N-desmethylolanzapine data are similar between the two reports but others, like clozapine / N-desmethylclozapine are widely different. We suspect that this is due to the much smaller sample numbers overall in the report by Kim et al. ${ }^{7}$ or an advantage of dilute and shoot analysis where all of the sample is injected onto the LC/MSMS instrument. ${ }^{16}$

The advantages of more prevalent metabolites are self-evident, providing greater effective sensitivity, easier analytical processes, and a better estimate of true negative samples. In addition to the metabolites mentioned above, we have reported improved metabolites for lurasidone ${ }^{17,18}$ and brexpiprazole. ${ }^{19}$ We concur with the authors that this could be an important way in which to monitor patient adherence versus blood testing. Most, if not all urine testing labs, can offer these tests in addition to conventional illicit drug testing (e.g., cocaine, amphetamines, PCP, marijuana, etc.). These suggestions are offered to improve antipsychotic testing even further for both clinical and forensic situations. Of course, these improvements in both analyte choice and sample processing (i.e., hydrolysis) will and should continue as newer antipsychotics and future antidepressants come to market.

Table I Comparison of the number of samples, the range, and the median values of antipsychotics and their metabolites from references ${ }^{7,8}$

\begin{tabular}{lllllll}
\hline Analyte & \multicolumn{1}{l}{$\mathbf{N}$} & Range & Median & $\mathbf{N}$ & Range & Median \\
\hline CZP & 5 & $95.9-288.3$ & 160.1 & 951 & $79-10702$ & 1226 \\
DMCZP & 5 & $316.1-1047.3$ & 589.9 & 777 & $277-28174$ & 3320 \\
OZP & 34 & $1.7-1882.8$ & 205 & 3539 & $31-3853$ & 327 \\
DMOZP & 34 & $1.5-1914.7$ & 168.3 & 3756 & $31-1940$ & 217 \\
QTP & 112 & $0.4-433.6$ & 15 & 6446 & $6.0-2286$ & 87 \\
NQTP & 112 & $3.9-2468$ & 164.1 & - & & \\
HOQTP & - & & & 7172 & $6.0-2047$ & 88 \\
HPD & 11 & $0.9-22.6$ & 3.5 & 2222 & $9.0-7758$ & 514 \\
RPD & 86 & $0.4-1117.4$ & 41 & 4405 & $6.0-1496$ & 92 \\
HORPD & 86 & $5.5-2255.2$ & 248.5 & 5691 & $10.0-3444$ & 857 \\
APZ & 32 & $0.6-557.6$ & 6 & 3173 & $5.0-150$ & 17 \\
DHAPZ & 32 & $1.9-35.0$ & 4.8 & 3581 & $5.0-87$ & 14 \\
OPC3373 & - & & 5151 & $47-5359$ & 782 \\
ZRD & 3 & $4.6-291.7$ & 24.4 & 1548 & $32-1803$ & 232 \\
ZRDSF & 3 & $3.5-10.1$ & 6.8 & - & &
\end{tabular}

Abbreviations: APZ, aripiprazole; DHAPZ, dehydroaripiprazole; CZP, clozapine; DMCZP, desmethylclozapine; QTP, quetiapine; NQTP, norquetiapine; HOQTP, 7-hydroxyquetiapine; HPD, haloperidol; RPD, risperidone; HORPD, hydroxyrisperidone; ZRD, ziprasidone; ZRDSF, ziprasidone sulfate; CPZ, chlorpromazine; HOCPZ, hydroxychlorpromazine; OZP, olanzapine; DMOZP, desmethylolanzapine

\section{Acknowledgments}

None.

\section{Conflicts of interest}

The author declares there is no conflict of interest.

\section{References}

1. Cohen CN, Collins G, Nucifora FC, et al. Clinical Consensus Recommendations for Urine Testing of Adherence to Antipsychotics Among People with Serious Mental Illness. Psychiatric Services. 2018;69:345-348
2. Fisher DS, Partridge SJ, Handley SA, et al. LC-MS/MS of Some Atypical Antipsychotics in Human Plasma, Serum, Oral Fluid, and Haemolysed Whole Blood. Forensic Sci International. 2013;229(1-3):145-150.

3. Miller J, Wehring $\mathrm{H}$, McMahon RP, et al. Urine Testing for Antipsychotics: A Pilot Trial for a Method to Determine Detection Levels. Hum Psychopharmacol. 2015;30(5):350-355

4. Application Note from Restek; restek.com/en/technical-literaturelibrary/articles/LC-MSMS-analysis-of-58-antipsychotics-andantidepressants-in-human-urine.

5. Dretchen KL, Miller R, McIntire GL, et al. Quantitative Levels of Aripiprazole and its Metabolites in Urine. Journal of Pharmacology and Clinical Toxicology. 2013;1(2):1014. 
6. McIntire GL. Methods of Monitoring for Adherence to Aripiprazole Therapy. U.S. Patent No., 20160003800, 2016.

7. Kim SY, Kim HS, Cheong JC. LC-MS-MS Determination of 25 Antipsychotic Drugs and Metabolites in Urine for Medication Compliance Monitoring. Journal of Analytical Toxicology. 2020;44:784-796.

8. Feng S, Enders JR, Cummings OT, et al. A Dilute and Shoot LC-MS/MS Method for Antipsychotics in Urine. Journal of Analytical Toxicology. 2020;44(4):331-338.

9. Cerlliant, O-049-1mL, Cerilliant.com/shopOnline/Product_cat_list. aspx?text=aripiprazole.

10. Strickland EC, Cummings OT, Morris AA. Impact of Monitoring Quetiapine Carboxylic Acid and Quetiapine Sulfoxide on Patient Positivity by Urine Drug Testing. Journal of Analytical Toxicology. 2013;40(8):687-693.

11.Cerlliant, O-049-1mL, Cerilliant.com/shopOnline/Product_cat_list. aspx? text=aripiprazole.

12. Baselt RC. Disposition of Toxic Drugs and Chemicals in Man. $11^{\text {th }}$ edition, Biomedical Publications: Seal Beach, CA, 2017. p. 455-457.

13. Strickland EC, Cummings OT, McIntire GL. Methods of Monitoring Adherence to Haloperidol Therapy. U.S. Patent Application No., 20170029866 , Feb 2, 2017.
14. Cummings OT, Strickland EC, Enders JR, et al. Impact of $\beta$-Glucuronidase Mediated Hydrolysis on Haldol® Urinalysis. Journal of Analytical Toxicology. 2018;42:214-219.

15. McIntire GL. Methods of Monitoring Adherence to Quetiapine Therapy. U.S. Patent No., 20160041192, February 11, 2016.

16. Enders JR, McIntire GL. A Dilute-and-Shoot LC-MS Method for Quantitating Opioids in Oral Fluid, Journal of Analytical Toxicology. 2015;39(8):662-667.

17. Strickland EC, Enders JR, McIntire GL. Determination of the Relative Prevalence of Lurasidone Metabolites in Urine Using Untargeted HRMS. Spectroscopy. 2019;17(2):8-15.

18. Enders JR, Strickland EC, McIntire GL. Methods of Monitoring Adherence to Lurasidone Therapy. U.S. Patent No 9606132, March 28, 2017.

19. Enders JR, Reddy SG, Strickland EC. Metabolites of Brexpiprazole in Human Urine. Clinical Mass Spectrometry. 2017;6:21-24. 